\title{
High Prevalence of blaCTX-M-1 Group Extended-Spectrum $\beta$-lactamase Genes in Escherichia coli Isolates From Tehran
}

\author{
Shahin Najar Peerayeh ${ }^{1,{ }^{*}}$, Majid Eslami ${ }^{1}$, Mojtaba Memariani ${ }^{1}$, Seyed Davar Siadat ${ }^{2}$ \\ ${ }^{1}$ Department of Bacteriology, Faculty of Medical Sciences, Tarbiat Modares University, Tehran, IR Iran \\ 2 Department of Hepatitis and AIDS, Pasteur Institute of Iran, Tehran, IR Iran \\ ${ }^{*}$ Corresponding author: Shahin Najar Peerayeh, Department of Bacteriology, Faculty of Medical Sciences, Tarbiat Modares University, Tehran, IR Iran. Tel: +98-2182883560, Fax: +98- \\ 2182884555, E-mail: najarp_s@modares.ac.ir.
}

Received: Jun 19, 2012; Revised: July 17, 2012; Accepted: August 08, 2012

\begin{abstract}
Background:The emerge of extended-spectrum $\beta$-lactamases(ESBLs)-producing Escherichia coli caused problems in antibiotic treatments. The more than 50 variants of CTX-M type ESBLs enzymes are available worldwide. Rapid and discriminative typing methods are essential for epidemiological analysis of clinical CTX-M-producing isolates.

Objectives: To assess the frequency and genetic diversity of extended-spectrum CTX-M $\beta$-lactamases in E. coli isolates from Tehran.

Materials and Methods: During 2009 - 2010, 200 non-duplicate clinical isolates of E. coli were collected from five hospitals in Tehran. Antibacterial susceptibility was determined using disk diffusion and MIC methods. ESBL production was confirmed by Combined Disk and MIC. CTX-M encoding genes were identified by PCR, and blaCTX-M-carrying isolates were genotyped by rep-PCR.

Results: A total of $140(70 \%)$ non-duplicate ESBL positive E. coli were determined. Imipenem and Amikacin were effective against 100\% and $85 \%$ of all tested isolates respectively. Co-resistance of ESBL positive isolates to Erythromycin, Tetracycline, Co-trimoxazole and Ciprofloxacin were found in $93 \%, 75 \%, 63 \%$ and $43 \%$ of the strains, respectively. The rate of cefotaxime and ceftazidime resistant isolates dramatically decreased from $72 \%$ to $8.5 \%$ and $39 \%$ to $4.5 \%$ respectively, in the presence of clavulanic acid. Eighty nine (44.5\%) E. coli isolates carried the blaCTX-M-1 group alleles. The levels of similarity of the rep-PCR fingerprints of blaCTX-M-1 isolates ranged from 40 to $90 \%$.

Conclusions: The rapid dissemination of non clonal multi-resistant CTX-M-1 producing E. coli isolates approved the need for further studies in our medical centers.
\end{abstract}

Keywords: Beta lactamase; E. coli; CTX-M-1; Rrep_PCR

\section{Background}

Plasmid-mediated extended-spectrum beta-lactamase (ESBL) enzymes-producing microorganisms are most common in both hospital and community settings. The extended-spectrum CTX-M $\beta$-lactamases are the most prevalent ESBLs worldwide, that first defined in Japan in 1986 (the enzyme was firstly named TOHO-1), and have been mainly associated with Enterobacteriasea, most prevalently in Escherichia coli, Klebsiella pneumoniae, Proteus mirabilis and Salmonella typhimurium (1). These enzymes are member of Ambler class A and classified in group 2be of the Bush, Jacoby and Medeiros classification and generally most active against cefotaxime and show little activity against ceftazidime and are inhibited by clavulanic acid and tazobactam $(2,3)$.

Phylogenetically, these enzymes have been classified into five major groups according to the amino acid similarities: the CTX-M-1cluster (CTX-M-1, -3, -10, -11, -12, -15, -28 and FEC-1), the CTX-M-2 cluster (CTX-M-2, -4, -5, -6, -7, -20, and TOHO-1), the CTX-M-8 cluster (CTX-M-8), the CTX-M-25 cluster (CTXM- 25 and -26), and the CTX-M-9 cluster (CTXM-9, -13, -14,-16, -17, -19, -21, -24, -27, and TOHO-2) $(4,5)$. The rapid dissemination of CTX-M enzymes is facilitated by plasmids, transposons, and integrons gene cassettes (6). Recently, prevalence of CTX-M-type extended-spectrum - $\beta$ lactamases have dramatically increased among Asian, African, European, and American E. coli clinical isolates (1, 2, 7). Therefore, rapid and discriminatory typing methods are essential for epidemiological analysis of CTX-M-producing isolates.

Little data on the type of CTX-M ESBL transmission is available at the regional level, and the genetic diversity of resistant isolates has not been reported until now. Rep-PCR DNA fingerprinting, is a rapid and simple typing method that relies on PCR amplification of the DNA between adjacent repetitive extragenic palindromic (rep) elements interspersed throughout the genome $(8,9)$.

Implication for health policy makers/practice/research/medical education

The identification of CTX-M producing clinical isolates besides rapid and discriminative typing methods are essential for epidemiological analysis of CTX-M producing clinical isolates. This study aims to determine the antimicrobial resistance and CTX-M ESBL production of E. coli isolates and also to determine their genetic diversity using PCR based fingerprinting techniques (rep-PCR). 


\section{Objectives}

This study aims to determine the antimicrobial resistance and E. coli CTX-M ESBL-producing isolates and also to determine their genetic diversity using PCR based fingerprinting techniques (rep-PCR).

\section{Materials and Methods}

\subsection{Clinical isolates}

During 2009 - 2010, 200 E. coli isolates were collected from 5 different teaching hospitals in Tehran. These isolates were obtained from blood cultures $(n=15)$, urine samples $(n=63)$, soft tissue specimens $(n=63)$, stool specimens $(n=38)$, and exudates samples $(n=21)$. The isolates were identified by conventional methods, and were stored in skimmed milk at $-70^{\circ} \mathrm{C}$ until required.

\subsection{Susceptibility Tests and Confirmation of ESBL Production}

Susceptibility testing was conducted by disk diffusion according to the guidelines of the Clinical and Laboratory Standards Institute (CLSI) (10). Fourteen Antimicrobial disks such as Cefoxitin, Ceftazidime, Cefotaxime, Cefepime, Aztreonam, Erythromycin, Gentamicin, Tetracycline, Co-trimoxazole, Amoxicillin, Amoxicillin-clavulanic acid, Imipenem, Amikacin, and Ciprofloxacin were used in this study. E. coli ATCC 25922 was used for quality control purposes in susceptibility testing. The ESBL phenotype was detected by combined disk methods using discs of Ceftazidime and Cefotaxime with and without clavulanic acid. The MIC of antibiotics were determined by agar dilution methods using Ceftazidime and Cefotaxime with and without clavulanic acid (10).

\subsection{Detection of the blaCTX-M Gene}

PCR template DNA was prepared using a boiling lysis method as previously described (11). The specific primers (Table 1) for diverse CTX-M groups (CTX-M-1, CTX-M-2, and CTX-M-9 groups) were used, as previously described $(12,13)$. Amplification reactions were performed in a total volume of $25 \mu \mathrm{L}$ of reaction mixture containing $5 \mu \mathrm{L}$ of 10× PCR buffer, $2.5 \mathrm{mM}$ Mgcl2, $200 \mu \mathrm{M}$ dNTPs, 1.25 units of Taq polymerase, $10 \mathrm{pmol}$ of each primer and $1 \mu \mathrm{L}$ of sample DNA. Amplification reactions were carried out in an Eppendorf thermal cycler (Eppendorf AG, Hamburg, Germany), with an initial denaturation $\left(4 \mathrm{~min}\right.$ at $94^{\circ} \mathrm{C}$ ) followed by 30 cycles of denaturation ( $60 \mathrm{~s}$ at $94^{\circ} \mathrm{C}$ ), annealing $\left(30 \mathrm{~s}\right.$ at $\left.55^{\circ} \mathrm{C}\right)$, and extension $\left(1 \mathrm{~min}\right.$ at $\left.72^{\circ} \mathrm{C}\right)$, with a single final extension of $5 \mathrm{~min}$ at $72^{\circ} \mathrm{C}$. DNA template from control clinical strains with well characterized CTX$M$ beta-lactamases (groups $1,2,9$ ) were used as the positive controls for PCR (14). The PCR products were analyzed on $1 \%$ agarose gels stained with ethidium bromide.

\begin{tabular}{lll}
\hline Table 1. Primers Used for Detection of blaCTX-M (12) & \\
\hline Target & Primer Sequence & Amplicon size, bp \\
\hline CTX-M1 (F) & F-GGT TAAAAAATC ACTGCG TC & 850 \\
& R-TTGGTGACGATTTTAGCCGC & \\
CTX-M2 & ATG ATG ACT CAG AGC ATT CG & 850 \\
& TGG GTT ACG ATT TTC GCC GC & 850 \\
CTX-M9 & ATG GTG ACA AAG AGA GTG CA & \\
& CCC TTC GGC GAT GAT TCT C
\end{tabular}

\section{4. rep-PCR}

DNA was extracted by using the Cinagen Mini kit (Cinagen Co. Iran). Repetitive extragenic palindromic sequence PCR (rep-PCR) have been described in detail in previous publications $(8,9)$. The primers REP1 (5-IIIGCGCCGICATCAGGC-3) and REP2 (5-ACGTCTTATCAGGCCTAC-3) was used to amplify putative REP-like elements in the genomic bacterial DNA. Amplification reactions were performed in a final volume of $25 \mu \mathrm{L}$ of $40 \mathrm{pmol}$ of each primers, $1.25 \mathrm{mmol} / \mathrm{L}$ of each four dNTPs, $3 \mathrm{U}$ Taq DNA polymerase, $1.5 \mathrm{mmol} / \mathrm{L} \mathrm{MgCl} 2,10 \mu \mathrm{L}$ of $10 \times$ PCR buffer and 10 ng of genomic DNA. PCR were carried out in an Eppendorf thermal cycler, with an initial denaturation (10 min at $94^{\circ} \mathrm{C}$ ) followed by 30 cycles of denaturation ( $1 \mathrm{~min}$ at $94^{\circ} \mathrm{C}$ ), annealing $\left(1 \mathrm{~min}\right.$ at $\left.40^{\circ} \mathrm{C}\right)$, extension $(6 \mathrm{~min}$ at $65^{\circ} \mathrm{C}$ ), and with a single final extension of $16 \mathrm{~min}$ at $65^{\circ} \mathrm{C}$. Aliquots $(10 \mu \mathrm{L})$ of each sample were subjected to electrophoresis on $2.0 \%$ agarose gel in Tris acetate-EDTA buffer at $50 \mathrm{~V}$ for $3 \mathrm{~h}$, stained with ethidium bromide, and visualized under UV light. In each run, rep- PCR was repeated for E. coli ATCC 25922, as internal control for the standardization of the PCR parameters.

Phoretix 1D and NTSYSpc software was used to make complete linkage dendrograms patterns. Bands Identification performed manually. The Dice similarity coefficient was used with position tolerance settings of $2.0 \%$. 
Najar Peerayeh Set al.

Rolling ball method was used to remove the background.

\section{Results}

\subsection{Antimicrobial Susceptibility and Detection of ESBL Production}

The results for the antibiotic resistance are shown in Table 2. Among the $\beta$-lactams tested, maximal resistance was noticed against amoxicillin (91\%) and amoxicillinclavulanic acid (85\%), while $39.5 \%$ and $72 \%$ of isolates were resistant to ceftazidime (CAZ) and cefotaxime (CTX) respectively. Resistance rate to other agents were $49 \%$ for Cefoxitin, 36\% for Cefepime, 39\% for Aztreonam, 94\% for Erythromycin, 36\% for Gentamicin, 58\% for Tetracycline, $\neg$ $57 \%$ for Co-trimoxazole , $15 \%$ for Amikacin, and 39\% for Ciprofloxacin. None of the isolates shows resistance to Imipenem.

Table 2. Antimicrobial Resistance Rate of E. coli Isolates (Quantity and Percentage Resistant) by Disk Diffusion Method

\begin{tabular}{lllllll}
\hline & Blood $(\mathbf{n}=\mathbf{1 5})$ & Urine $(\mathbf{n}=\mathbf{6 3})$ & Stool $(\mathbf{n}=\mathbf{3 8})$ & Soft tissue $(\mathbf{n}=\mathbf{6 3})$ & Exudates $(\mathbf{n}=\mathbf{2 1})$ & Total $(\mathbf{n = 2 0 0})$ \\
\hline Amoxicillin & $12(8 \%)$ & $58(92 \%)$ & $35(92.1 \%)$ & $60(95.3 \%)$ & $18(85.7 \%)$ & $183(91.5 \%)$ \\
$\begin{array}{l}\text { Amoxicillin- } \\
\text { clavulanic acid }\end{array}$ & $11(73.3 \%)$ & $50(79.3 \%)$ & $35(92.1 \%)$ & $56(88.8 \%)$ & $18(85.7 \%)$ & $170(85 \%)$ \\
Amikacin & $1(6.6 \%)$ & $15(23.8 \%)$ & $2(5.2 \%)$ & $10(15.8 \%)$ & $3(14.2 \%)$ & $31(15.2 \%)$ \\
Aztreonam & $4(26.6 \%)$ & $23(36.5 \%)$ & $10(26.3 \%)$ & $33(52.3 \%)$ & $9(42.8 \%)$ & $79(39.5 \%)$ \\
Ceftazidime & $7(46.6 \%)$ & $22(34.9 \%)$ & $12(31.5 \%)$ & $28(44.4 \%)$ & $10(47.6 \%)$ & $79(39.5 \%)$ \\
Cefotaxime & $10(66.6 \%)$ & $42(66.6 \%)$ & $24(63.1 \%)$ & $49(77.7 \%)$ & $19(90.4 \%)$ & $144(72 \%)$ \\
Cefoxitin & $3(20 \%)$ & $26(41.2 \%)$ & $19(50 \%)$ & $38(60.3 \%)$ & $7(33.3 \%)$ & $93(46.2 \%)$ \\
Cefepime & $3(20 \%)$ & $22(34.9 \%)$ & $35(92.1 \%)$ & $29(46 \%)$ & $6(28.5 \%)$ & $72(36 \%)$ \\
co-trimoxazole & $4(26.6 \%)$ & $39(61.9 \%)$ & $22(57 / 8 \%)$ & $37(58.7 \%)$ & $12(57.1 \%)$ & $114(57 \%)$ \\
Ciprofloxacin & $6(40 \%)$ & $23(36.5 \%)$ & $9(23.6 \%)$ & $33(52.3 \%)$ & $7(33.3 \%)$ & $78(39 \%)$ \\
Erythromycin & $11(73.3 \%)$ & $60(95.2 \%)$ & $34(89.4 \%)$ & $63(100 \%)$ & $20(95.2 \%)$ & $188(94 \%)$ \\
Gentamicin & $6(40 \%)$ & $18(28.5 \%)$ & $3(7.8 \%)$ & $32(50.7 \%)$ & $14(66.6 \%)$ & $73(36.2 \%)$ \\
Imipenem & 0 & 0 & 0 & 0 & 0 & 0
\end{tabular}

A total of 140 (70\%) non-duplicate clinical isolates of ESBL positive E. coli were detected based on their phenotype using cefotaxime/clavulanate and ceftazidime/clavulanate. The difference in inhibition zones of $\beta$-lactam disks with and without clavulanic acid was $5-10 \mathrm{~mm}$.

The MICs of all isolate were determined for CTX and CTZ with and without inhibitor (Table 3). A total of 144 (72\%) isolates were resistant (MIC $\geq 4 \mathrm{mg} / \mathrm{L}$ ) to CTX, and $42(29 \%)$ of resistant isolates expressed high $\mathrm{MIC}(256 \mathrm{mg} / \mathrm{L} \leq)$. The
MIC of CTZ for 78 (48\%) of isolates were resistant (MIC $\geq$ $16 \mathrm{mg} / \mathrm{L})$, and 11 (14\%) of resistant isolates expressed high $\operatorname{MIC}(256 \leq)$.

The phenotypic ESBL agar dilution confirmatory test was positive, and the rate of CTX and CTZ resistant isolates dramatically decreased from $72 \%$ to $8.5 \%$ and $39 \%$ to $4.5 \%$ respectively, in the presence of clavulanic acid (Table 3).

Table 3: MICs of E. coli Isolates for CAZ and CTX With and Without Clavulanate

\begin{tabular}{|c|c|c|c|c|c|c|c|c|c|}
\hline$\mu \mathrm{g} / \mathbf{m L}$ & $1 \geq$ & 2 & 4 & 8 & $16-32$ & $64-128$ & $256-512$ & $512 \leq$ & Resistant \\
\hline CTX & 50 & 6 & 23 & 24 & 21 & 34 & 21 & 21 & 144 \\
\hline CTX-CA ${ }^{\mathrm{a}}$ & 179 & 4 & 7 & 8 & 0 & 0 & 0 & 2 & 17 \\
\hline $\mathbf{C A Z}^{\mathrm{a}}$ & 53 & 6 & 24 & 39 & 54 & 13 & 1 & 10 & 78 \\
\hline CAZ-CA & 76 & 46 & 32 & 37 & 3 & 4 & 1 & 1 & 9 \\
\hline
\end{tabular}

a Abbreviations: CTX; Cefotaxime, CAZ; Ceftazidime, CA; Clavulanic acid

\subsection{Detection of blaCTX-M Gene}

PCR was performed for all CTX resistant isolates according to results obtained by phenotypic tests. Eighty nine
(61.8\%) of the 144 CTX resistant E. coli isolates carried the blaCTX-M-1 group alleles (Figure 1). None of the isolates were positive for CTX-M groups 2 and 9. 


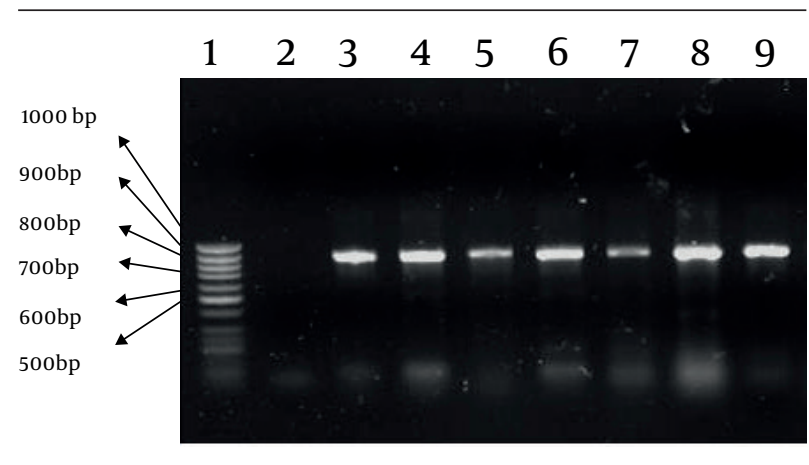

Figure 1.Lanes:1.100bpladder, Lane 2. Negative control(E. coli ATCC25922), Lane 3. Positive control blaCTXM-1,Lane 4 to 9. Clinical isolates

The majority of blaCTX-M-1-carrying isolates were recovered from urine (33.7\%) and tissue (32.5\%), followed by 18
(20.2\%) from stool, and 9 (10.1\%) from exudates and 3 (3.3\%) from blood. The resistance of blaCTX-M-1 -carrying E. coli isolates to antibiotics were $93.2 \%$ for amoxicillin and amoxicillin-clavulanic acid, $70.7 \%$ to ceftazidime, $68.5 \%$ cefoxitin, $65.1 \%$ to cefepime, $86.5 \%$ to Aztreonam, $94.3 \%$ to Erythromycin, $38.2 \%$ to Gentamicin, $75.2 \%$ to Tetracycline, $67.4 \%$ to Co-trimoxazole, $14.6 \%$ to Amikacin, and $43.8 \%$ to Ciprofloxacin. No imipenem resistance was detected.

The MIC of CTX for 38 (42\%) blaCTX-M-1 -carrying E. coli isolates was high $(256 \leq)$. While the MIC of $7(7.8 \%)$ of blaCTX-M-1 -carrying isolates were $4-8 \mu \mathrm{g} / \mathrm{mL}$. The MIC of CTX for all blaCTX-M-1 -carrying isolates was reduced by 4 - 7 doubling dilutions in the presence of clavulanic acid. The blaCTX-M-1 -carrying isolates were also resistant to CAZ, and only 11 isolates were susceptible.

Figure 2. rep-PCR Analysis for blaCTX-M1 Isolates.

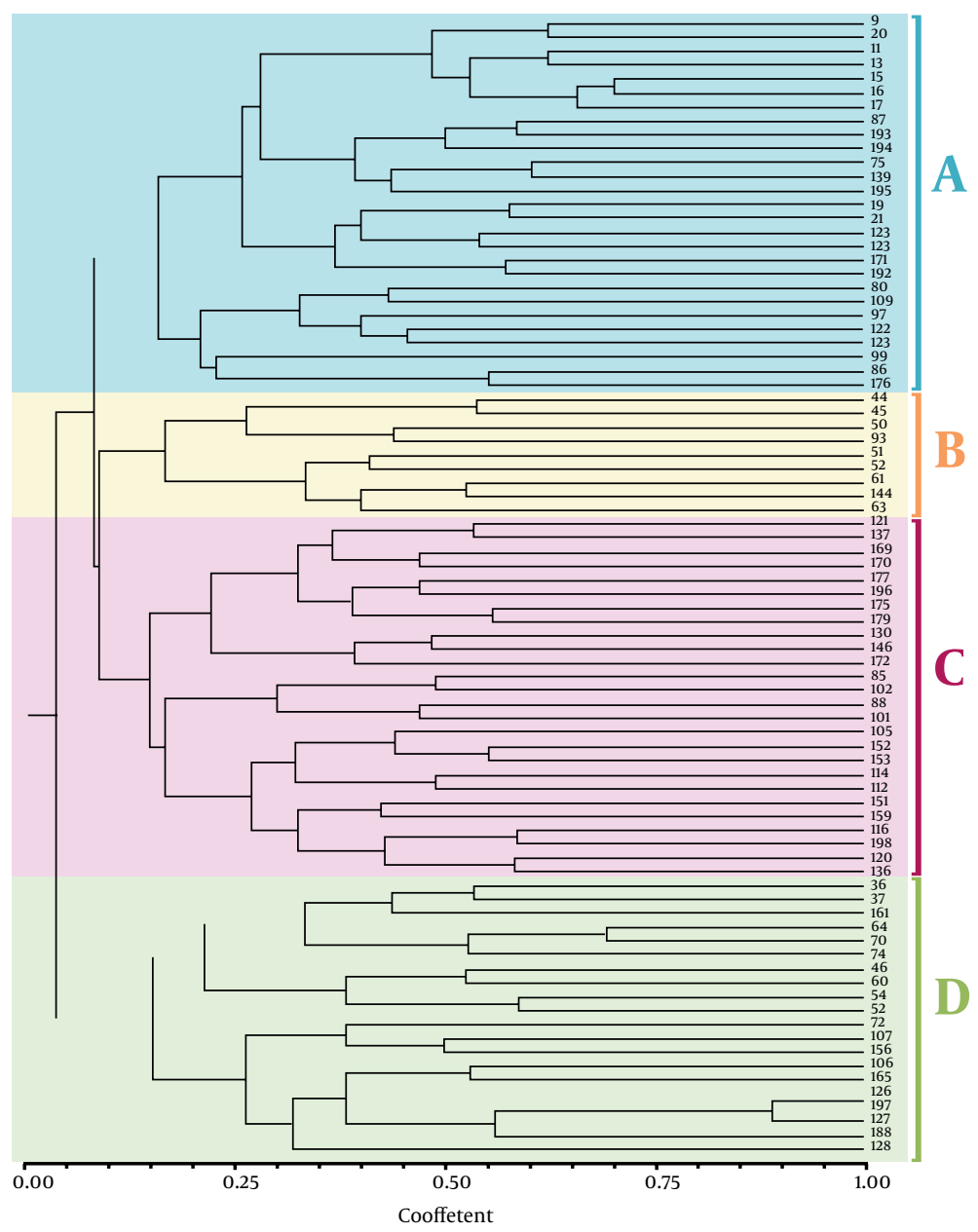

Phoretix1D and NTSYSpc software was used to make Complete Linkage dendrograms of fragment patterns. The blaCTX-M1 isolates classified into four clusters. 


\section{3. rep-PCR}

Molecular typing with rep-PCR was used to define the clonal patterns of 89 CTX-M1 isolates. There were no common rep-PCR fingerprint patterns that provided an average to differentiate CTX-M1 isolates. However, these isolates included 4 different clusters (Figure 2). The genetic fingerprints of isolates obtained different sources such as blood, urine, and stool were similar, and differences were found between isolates obtained from the same samples. There was also considerable genetic diversity among isolates collected from the same hospital. The levels of similarity of the rep-PCR fingerprints of isolates ranged from 40 to $90 \%$.

\section{Discussion}

Multidrug resistant isolates of E. coli have been increasing worldwide, and is associated with the acquisition of mobile element-encoded $\beta$-lactamases. The production of CTX-M $\beta$-lactamases is an important mechanism of resistance to oxyimino-cephalosporins in this microorganism. This family of plasmid-mediated ESBLs of Ambler class A that has been detected mainly in Asian countries, Europe, and America, (2,12-14). In this study, 140 isolates (70\%) showed the ESBL phenotype by combined disk and Agar dilution methods. In a study conducted by Mirzaee, $56 \%$ of $E$. coli isolates from three university hospitals of Tehran revealed ESBL phenotype, which is less than our findings (15). Obviously, our results were analyzed based on the new 2010 CLSI interpretative criteria (10). A high incidence of ESBL-producers amongst E. coli was reported from India (79\%), Pakistan (64\%) and Kuwait (77.9\%) (1618). These studies indicate a high incidence of ESBL-producers amongst E. coli isolates in Asian countries.

In this study, a total of $63.5 \%$ of ESBL producers isolates carried blaCTX-M-1, this subgroup of blaCTX-M is prevalent in our country $(15,19)$. The blaCTX-M-1 has detected as the most common type of blaCTX-M from Asia, Europe, North and South America among multidrug-resistant $E$. coli $(17,20-22)$. A high level resistance to $\beta$-lactam, as well as Erythromycin, Tetracycline, and Co-trimoxazole, was observed among our blaCTX-M-1 carrying isolates. However, aminoglycosides such as Amikacin and to some extent, Gentamicin was showed the active antibacterial effects. Imipenem was the most effective antibiotic against all ESBL- producing E. coli.

Analysis of the rep-PCR typing of the bla CTX-M-1 -carrying isolates showed that there is no evidence of any clonality within a single hospital or clonal spread across hospitals. The heterogeneous natures of these enzymes were reported by $\mathrm{Al}$ Hashem et al. (16), and Khanna et al. (23). The rapid dissemination of non-clonal multi-resistant CTX-M-1 producing E. coli isolates indicates the need for further investigations in medical centers.

\section{Acknowledgements}

The authors would like to thank Dr. Mohsen Mirzaee for providing isolates carrying bla CTX-M-1 genes.

\section{Authors' Contribution}

None declared.

\section{Financial Disclosure}

None to declare

\section{Funding/Support}

This work was supported by grants from faculty of Medical Science, Tarbiat Modares University (Tehran, Iran) and the Iran National Science Foundation (Tehran, Iran).

\section{References}

1. Bonnet R. Growing group of extended-spectrum beta-lactamases: the CTX-M enzymes. Antimicrob Agents Chemother. 2004;48(1):1-14

2. Canton R, Coque TM. The CTX-M beta-lactamase pandemic. Cur Opin Microbiol.2006;9(5):466-75.

3. Tzouvelekis LS, Tzelepi E, Tassios PT, Legakis NJ. CTX-M-type $\beta$-lactamases: an emerging group of extended-spectrum enzymes. Int J Antimicrob Agents. 2000;14(2):137-142.

4. Poirel L, Kampfer P, Nordmann P. Chromosome-encoded Ambler class A beta-lactamase of Kluyvera georgiana, a probable progenitor of a subgroup of CTX-M extended-spectrum beta-lactamases. Antimicrob Agents Chemother. 2002;46(12):4038-40.

5. Soge OO, Adeniyi BA, Roberts MC. New antibiotic resistance genes associated with CTX-M plasmids from uropathogenic Nigerian Klebsiella pneumoniae. J Antimicrob Chemother. 2006;58(5):104853.

6. Eckert C, Gautier V, Arlet G. DNA sequence analysis of the genetic environment of various blaCTX-M genes. J Antimicrob Chemother. 2006;57(1):14-23.

7. Rossolini GM, D'Andrea MM, Mugnaioli C. The spread of CTX-Mtype extended-spectrum beta-lactamases. Clin Microbiol Infect. 2008;14 Suppl 1:33-41.

8. Olive DM, Bean P. Principles and applications of methods for DNA-based typing of microbial organisms. J Clin Microbiol. 1999;37(6):1661-9.

9. Versalovic J, Koeuth T, Lupski JR. Distribution of repetitive DNA sequences in eubacteria and application to fingerprinting of bacterial genomes. Nucleic Acids Res. 1991;19(24):6823-31.

10. Wikler MA. Performance Standards for Antimicrobial Susceptibility Testing: Twentieth Informational Supplement. Clinical and Laboratory Standards Institute; 2010.

11. Eckert C, Gautier V, Saladin-Allard M, Hidri N, Verdet C, OuldHocine Z, et al. Dissemination of CTX-M-type beta-lactamases among clinical isolates of Enterobacteriaceae in Paris, France. Antimicrob Agents Chemother. 2004;48(4):1249-55.

12. Castanheira M, Mendes RE, Rhomberg PR, Jones RN. Rapid emergence of blaCTX-M among Enterobacteriaceae in U.S. Medical Centers: molecular evaluation from the MYSTIC Program (2007). Microb Drug Resist. 2008;14(3):211-6.

13. Chanawong A, M'Zali FH, Heritage J, Xiong JH, Hawkey PM. Three cefotaximases, CTX-M-9, CTX-M-13, and CTX-M-14, among Enterobacteriaceae in the People's Republic of China.Antimicrob Agents Chemother. 2002;46(3):630-7.

14. Mirzaee M, Owlia P, Mansouri S. Distribution of CTX-M $\beta$-lactamase genes among Escherichia coli strains isolated from patients in Iran. Lab Medicine. 2009;40(12):724-727.

15. Chaudhuri BN, Rodrigues C, Balaji V, Iyer R, Sekar U, Wattal C, et 
al. Incidence of ESBL producers amongst Gram-negative bacilli isolated from intra-abdominal infections across India (based on SMART study, 2007 data). JAssoc Physicians India. 2011;59:287-92.

16. Al Hashem G, Al Sweih N, Jamal W, Rotimi VO. Sequence analysis of bla(CTX-M) genes carried by clinically significant Escherichia coli isolates in Kuwait hospitals. Med Princ Pract. 2011;20(3):213-9.

17. Feizabadi MM, Delfani S, Raji N, Majnooni A, Aligholi M, Shah cheraghi F, et al. Distribution of bla(TEM), bla(SHV), bla(CTX-M) genes among clinical isolates of Klebsiella pneumoniae at Labbafinejad Hospital, Tehran, Iran. Microb Drug Resist. 2010;16(1):4953.

18. Hussain M, Hasan F, Shah AA, Hameed A, Jung M, Rayamajhi N, et al. Prevalence of Class A and AmpC b-Lactamases in Clinical Escherichia coli Isolates from Pakistan Institute of Medical Science, Islamabad, Pakistan.Jpn.J. Infect. Dis. 2011;64:249-252.

19. Boyd DA, Tyler S, Christianson S, McGeer A, Muller MP, Willey $\mathrm{BM}$, et al. Complete nucleotide sequence of a 92-kilobase plasmid harboring the CTX-M-15 extended-spectrum beta-lactamase involved in an outbreak in long-term-care facilities in Toronto, Canada. Antimicrob Agents Chemother. 2004;48(10):3758-64.

20. Lewis JS, 2nd, Herrera M, Wickes B, Patterson JE, Jorgensen JH. First report of the emergence of CTX-M-type extended-spectrum beta-lactamases (ESBLs) as the predominant ESBL isolated in a U.S. health care system. Antimicrob Agents Chemother. 2007;51(11):4015-21.

21. Radice M, Power P, Di Conza J, Gutkind G. Early dissemination of CTX-M-derived enzymes in South America. Antimicrob Agents Chemother. 2002;46(2):602-604.

22. Woodford N, Ward ME, Kaufmann ME, Turton J, Fagan EJ, James D, et al. Community and hospital spread of Escherichia coli producing CTX-M extended-spectrum beta-lactamases in the UK. $J$ Antimicrob Chemother. 2004;54(4):735-43.

23. Khanna N, Boyes J, Lansdell PM, Hamouda A, Amyes SG. Molecular epidemiology and antimicrobial resistance pattern of extended-spectrum-beta-lactamase-producing Enterobacteriaceae in Glasgow, Scotland. J Antimicrob Chemother. 2012;67(3):573-7. 